\title{
Trends in Glaucoma Medication Expenditures under Universal Health Coverage: A National Population-Based Longitudinal Survey in Taiwan
}

\author{
Shin-Lin Chiu, ${ }^{1,2}$ Chiao-Lee Chu, ${ }^{2}$ Chih-Hsin Muo, ${ }^{3}$ Chiu-Liang Chen, ${ }^{1,4}$ and Shou-Jen Lan ${ }^{2}$ \\ ${ }^{1}$ Department of Ophthalmology, Changhua Christian Hospital, 135 Nanxiao Street, Changhua City, Changhua County 500, Taiwan \\ ${ }^{2}$ Department of Healthcare Administration Management, Asia University, 500 Lioufeng Road, Wufeng, Taichung 41354, Taiwan \\ ${ }^{3}$ Management Office for Health Data, China Medical University Hospital, 2 Yude Road, Taichung 40447, Taiwan \\ ${ }^{4}$ Department of Orthopedics, Changhua Christian Hospital, 135 Nanxiao Street, Changhua City, Changhua County 500, Taiwan
}

Correspondence should be addressed to Chiao-Lee Chu; chiaoleechu@gmail.com

Received 29 March 2015; Revised 20 May 2015; Accepted 25 May 2015

Academic Editor: Van C. Lansingh

Copyright (C) 2015 Shin-Lin Chiu et al. This is an open access article distributed under the Creative Commons Attribution License, which permits unrestricted use, distribution, and reproduction in any medium, provided the original work is properly cited.

\begin{abstract}
Medical care in Taiwan is well known for its low cost, high efficiency, high quality, excellent medical accessibility, and high equity. We investigate the trends in medication expenditures for glaucoma from 1997 to 2010 . The results show that higher medical expenditures were incurred by patients who were aged $\geq 40$ years, male patients, and patients in the highest salary population whereas lower medical expenditures were incurred by blue-collar workers. The medications with the most significant increases in expenditure were prostaglandin analogs (PGAs), $\alpha$-agonists, and fixed combinations, whereas the medications with the most significant decreases in expenditure were $\beta$-blockers and cholinergic agonists. The number of trabeculectomies shows two downward break points in 1999 and 2000 when PGAs were listed and were reimbursed. These results suggest socioeconomic disparities in glaucoma care, as well as understanding of the changes in the expenditure of glaucoma medications under such universal health insurance coverage system.
\end{abstract}

\section{Introduction}

Glaucoma is the second leading cause of blindness, affecting approximately 60.5 million people worldwide or about $2.6 \%$ of the population over the age of 40 [1]. Although the most prevalent form of glaucoma in western countries and in many other parts of the world is open-angle glaucoma (OAG) [1], angle-closure glaucoma (ACG) is the most common type of glaucoma among the Han Chinese [2].

Blindness due to glaucoma may be preventable if patients are given adequate treatment. Factors associated with the development of glaucoma include increased intraocular pressure (IOP), higher cup-to-disc ratio, aging, thinner central corneal thickness, family history of glaucoma, myopia, and chronic and systemic diseases such as diabetes $[3,4]$. Most ophthalmologists treat glaucoma by lowering the IOP using one of three modalities-topical antiglaucoma drugs, laser treatment, or glaucoma surgery. Types of topical medication include $\beta$-blockers, $\alpha$-agonists, prostaglandin analogs
(PAGs), carbonic anhydrase inhibitors (CAIs), cholinergic agonists, and adrenergic agonists [5]. Because some patients require multiple daily dosing, fixed combination eye drops have been developed to enhance and reinforce patient compliance [6].

Recent studies have shown that glaucoma treatment in some developed countries causes a significant financial burden on the health care system [7-9]. In this study, we used the National Longitudinal Health Insurance Database 2000 (LHID2000) to examine trends in glaucoma medication expenditure in Taiwan from 1997 to 2010. The Taiwan National Health Insurance (NHI) program is a mandatory single-payer health insurance system under which all residents are covered. The NHI program has been in existence since 1995 and by the end of 2010 the coverage rate was $99 \%$ of Taiwan's population of 23.1 million. Health spending accounted for $6.9 \%$ of GDP in Taiwan. Medical care in Taiwan is well known for its low cost, convenience, high efficiency, high quality, and excellent medical accessibility 
[10-12]. The nationwide population-based dataset provides an opportunity to explore the trends in glaucoma medication expenditure.

\section{Materials and Methods}

2.1. Database. This study was designed as a population-wide retrospective review using the Taiwan National Health Insurance Research Database (NHIRD). The NHIRD contains a large number of computerized records including registration files, medication and treatment regimens, and information on surgery. Claim reimbursement data from patients since 1999 are provided to researchers in an electronically encrypted form. Data contained in the LHID2000 are randomly selected from one million subjects from the NHIRD and are made available for research purposes [13]. Data from the LHID2000 used in this study included patient identification numbers, gender and age, monthly salary, occupation type, diagnostic data, antiglaucoma drug codes, and surgery codes. Diseases are defined in accordance with A codes (A230) before 2000 and International Classification of Diseases, 9th Revision, Clinical Modification (ICD-9-CM365), after 2000.

The study received approval from the Ethics Committee of the Institutional Review Board of the Changhua Christian Hospital and was conducted in accordance with the tenants of the Declaration of Helsinki.

2.2. Study Sample. This study group comprised all patients with glaucoma during the period 1997 to 2010. Topical glaucoma medications included PGAs, $\beta$-blockers, $\alpha$ agonists, CAIs, cholinergic agonist, $\beta$-blockers/CAIs fixed combinations, $\beta$-blockers $/ \alpha$-agonists fixed combination, $\beta$ blockers/PGAs fixed combinations, and adrenergic agonists. Data on frequency of trabeculectomy were also collected.

2.3. Statistical Analyses. The annual expenditures for glaucoma patients were adjusted for inflation as of 2011. The trend test for expenditure was analyzed with linear regression weighted with the inverse of squares residual. The associations between increase and decline in medication costs were analyzed with Pearson correlation. The trend test for frequency of trabeculectomy was analyzed with the Chow test [14]. We also assessed the effect of demographic factors on glaucoma expenditure using a generalized estimating equation regression model (GEE). A $P$ value $<0.05$ was considered to indicate statistical significance; all tests were two-tailed. All statistical analyses were conducted using the statistical package SAS for Windows (Version 9.2).

\section{Results}

The number of beneficiaries included in the LHID2000 sample declined from 916,626 persons in 1997 to 859,913 persons in 2010. The number of patients receiving antiglaucoma drugs increased from 3105 in 1997 to 7033 in 2010 (Table 1).

3.1. Overall Costs. After adjusting for inflation, the total annual medical expenditures increased from $\$ 0.21$ million in

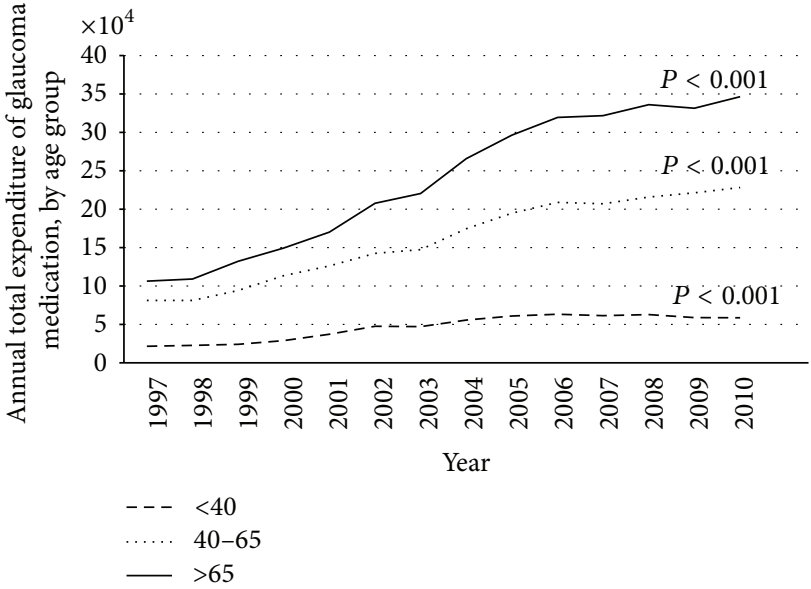

FIGURE 1: Plot of annual total expenditure of glaucoma medication, by age group.

1997 to $\$ 0.63$ million in 2010 (slope $=\$ 37,618 /$ year, $P<0.001$ ) (Table 1). The increase in total expenditures was remarkable in both genders, every age group, every income group, and every occupation group $(P<0.05)$. Annual glaucoma medication expenditures for men were higher than those for women after 2000. The annual glaucoma medication expenditures were most prominent in the age group $\geq 65$ years, followed by the age group 40-64 years and the age group $<40$ years (Figure 1). The lowest income group spent more money on antiglaucoma medications than other income groups. The white-collar occupation group spent more money on medications than the other occupation groups.

3.2. Per Capita Expenditure Costs. The mean medication expenditure per person increased from $\$ 67.3$ in 1997 to $\$ 90$ in 2010 after adjusting for inflation (slope $=\$ 2.8 /$ year, $P<$ 0.001 ) (Table 1). The mean cost per capita increased year on year from 1997 to 2006 and then decreased gradually after 2007. The trend in mean medication expenditures per person was similar in each category (gender, age, income, and occupation group). Estimates from the GEE conducted to determine demographic variables associated with the increase in glaucoma medication expenditure indicate that patients $\geq 40$ years incurred higher costs for medication than patients under the age of 40 years $(P<0.001)$. In the same analysis, men incurred higher costs than women $(P<0.05)$, populations with higher income incurred higher costs for medications than populations with lower income $(P<0.05)$, glaucoma medication expenditures increased year on year $(P<0.001)$, and blue-collar workers had lower expenditures than the other types of workers $(P<0.001)$ (Table 2$)$.

\subsection{The Changes of Expenditure between Different Glaucoma} Medications. The annual expenditures for most classes of glaucoma medications increased during the study period, except for $\beta$-blockers (slope $=-\$ 10,152 /$ year, $P<0.001$ ) and cholinergic agonists (slope $=-\$ 432 /$ year, $P<0.001)$ (Table 1$)$. The decrease in expenditure for $\beta$-blockers was associated 


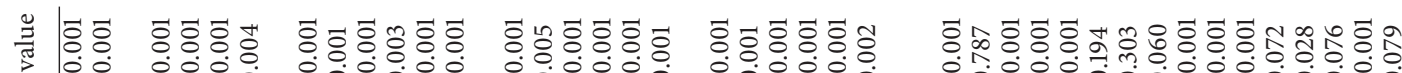
Q

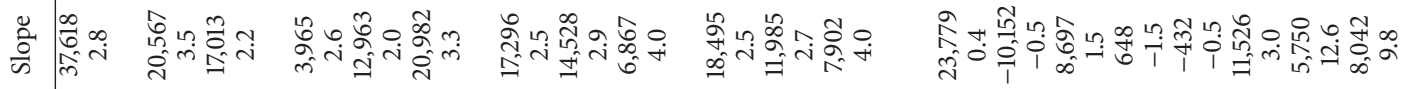

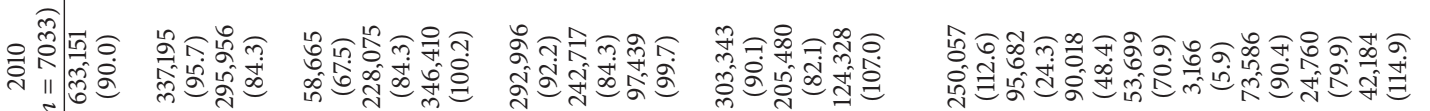

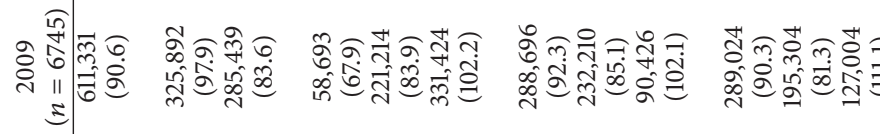

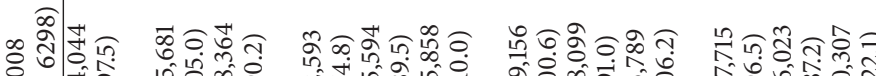

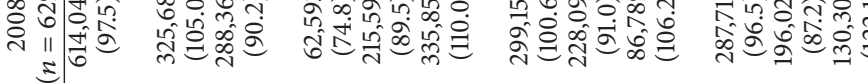

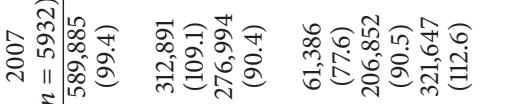

ind

:ล大亏

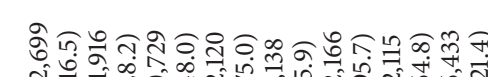

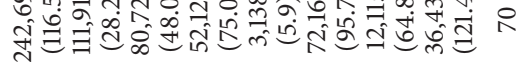

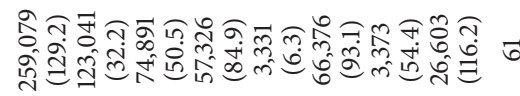
总商

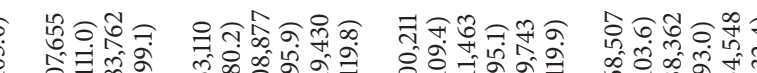

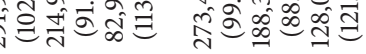

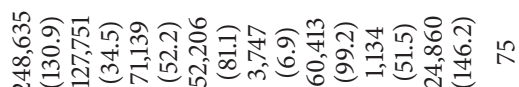

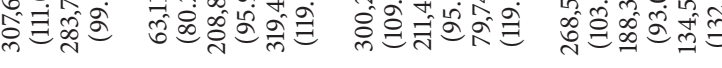

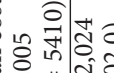

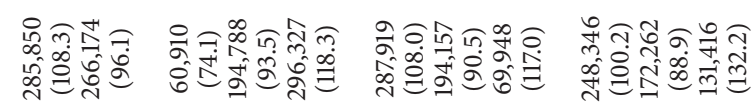

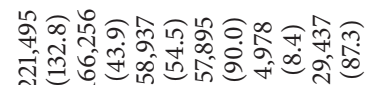

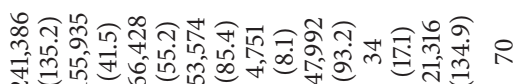

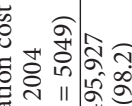

총ํำ

过定

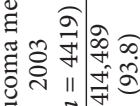

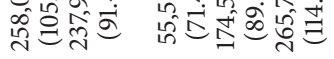

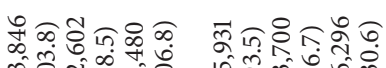

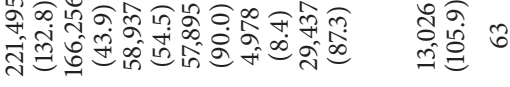

일

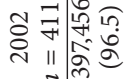

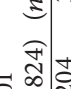

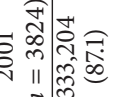

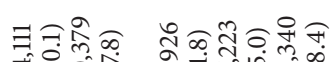

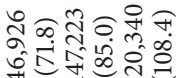

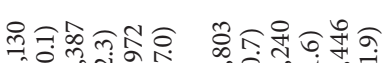

공유

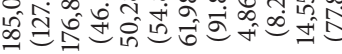

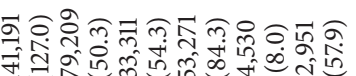

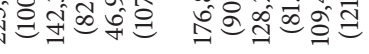

낙으으

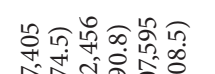

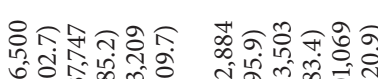

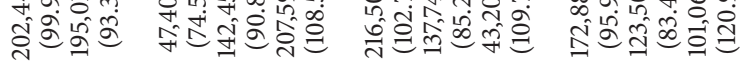

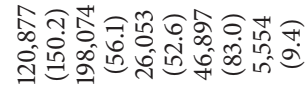

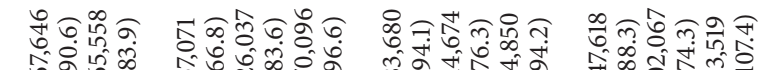
5

के

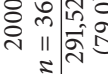

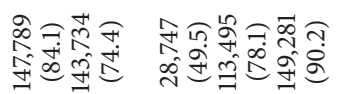

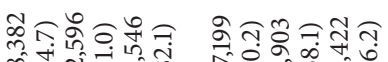

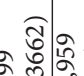

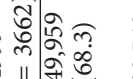

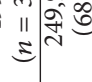

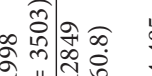

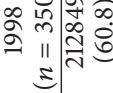

fic

구응

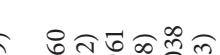
象运

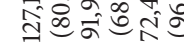

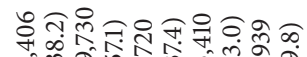

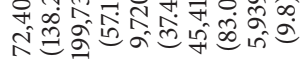

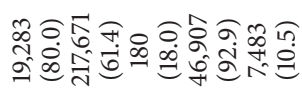

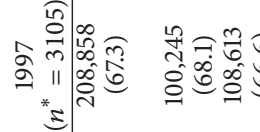

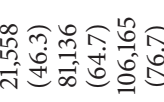

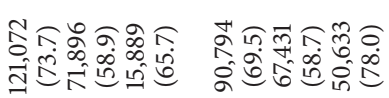

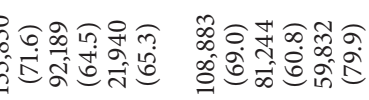

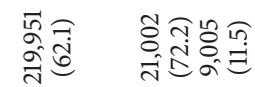

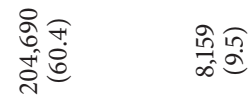

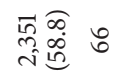

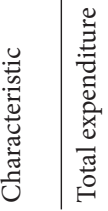

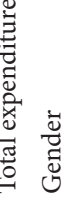

बै

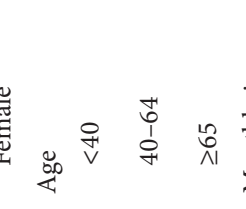

:

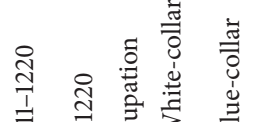

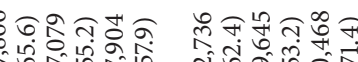

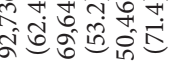

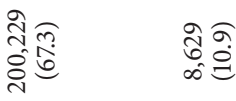

เ 
TABLE 2: The results of generalized estimating equations regression model (USD/year).

\begin{tabular}{lccc}
\hline & Estimate & SE & $P$ value \\
\hline Intercept & 33.4 & 1.5 & $<0.001$ \\
Age, year (versus $<40)$ & & & \\
$\quad 40-64$ & 10.4 & 1.4 & $<0.001$ \\
$\quad 185$ & 2.5 & 1.5 & $<0.001$ \\
$\quad$ Gender (versus female) & & 1.1 & 0.041 \\
Income, USD (versus $\leq 610)$ & -3.2 & 1.3 & 0.015 \\
$\quad 611-1220$ & 6.7 & 2.0 & $<0.001$ \\
$\quad>1220$ & & & \\
Occupation (versus white-collar & & & \\
workers) & -6.7 & 1.3 & $<0.001$ \\
$\quad$ Blue-collar workers & 6.8 & 1.6 & $<0.001$ \\
$\quad$ Other types of workers & 1.0 & 0.1 & $<0.001$ \\
Year & & & \\
\hline
\end{tabular}

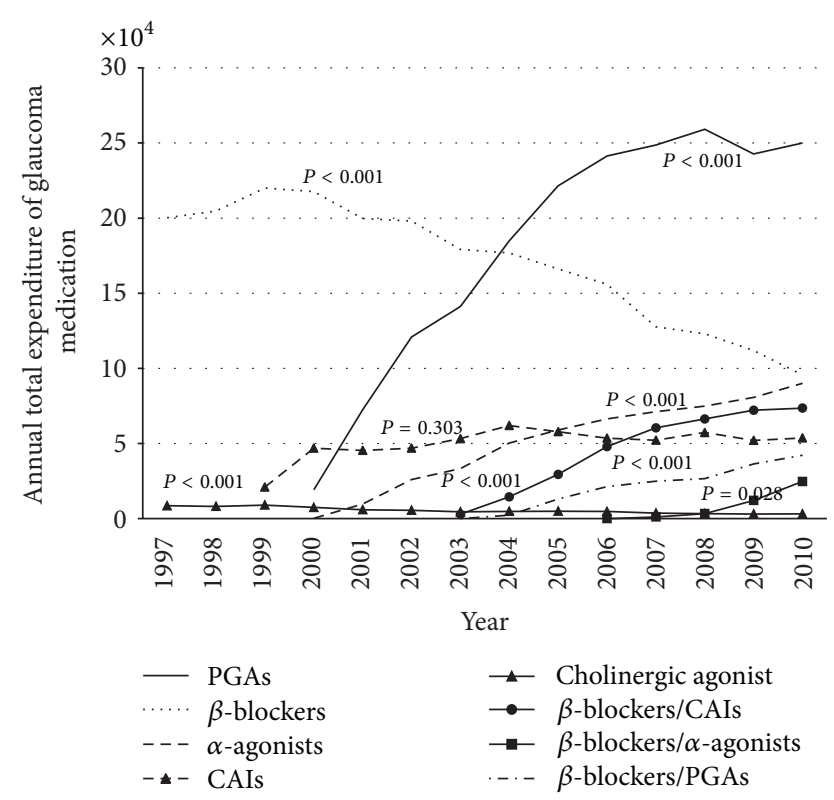

Figure 2: Plot of total expenditure of glaucoma medication, by medication class.

with the administration of PGAs $(P=0.002)$, and $\alpha$-agonists $(P<0.001)$. A significant increase in expenditures on PGAs (slope $=\$ 23,779 /$ year, $P<0.001$ ) was also noted. Medications containing PGAs accounted for $46 \%$ of the total glaucoma medication expenditure in 2010 (Table 1) (Figure 2).

We also found a decreasing trend in per capita medication expenditures for $\beta$-blockers (slope $=-\$ 0.5 /$ year, $P<0.001$ ) and cholinergic agonists (slope $=-\$ 0.5$ /year, $P<0.001$ ). However, the trends for other glaucoma medications were not significant (Table 1).

3.4. The Change of Trabeculectomy Number during the Study Period. The numbers of trabeculectomies performed during the study period are shown in Table 1. During the period 1997-2010, we found that the frequency of trabeculectomy had two break points, one in the year 1999 and the other in the year 2000 based on the Chow test [14].

\section{Discussion}

During the study period, the total expenditures for glaucoma medications significantly increased by 3.03-fold because of an increase in patient numbers and an increase in mean medication expenditures per person. The increase in patient numbers may be related to the early diagnosis due to advanced diagnostic modalities (e.g., optical coherence tomography), more accurate diagnosis, overdiagnosis, aging population, or good medical accessibility in Taiwan $[10,11$, 15]. The increase in mean medication expenditure per person may be associated with the administration of PGAs and more aggressive glaucoma treatment [5]. The rising cost of glaucoma drugs after PGAs launching also occurred in Ireland, Scotland, Australia, Denmark, and France [16].

As seen in Table 2, mean glaucoma medication expenditures increased markedly after the NHI system began covering treatment with PGAs in 2000. The reduction of expenditure per capita after 2007 and the mean medication cost in Taiwan was much lower than in Denmark and the United States [8,9] reflecting the success of bargaining medication cost and prescription policy (beta-blocker should be first-line drug) by Taiwan NHI. However, the increasing total medication expenditure imaged that the cost down policy could not offset the growing medical demand.

In this study, expenditures for glaucoma medications were significantly higher for men than for women, whereas in the USA the opposite is true [8]. The possible reasons are differences in knowledge of health issues between genders in different societies and the higher prevalence of ACG in Taiwan $[2,17]$. Women are at higher risk of ACG [18] that can be treated with laser iridotomy or cataract surgery rather than glaucoma medications. Cataract surgery is readily available in Taiwan and the procedure reduces the likelihood of developing the disease $[13,19]$.

It is not surprising that glaucoma medical expenditures were highest among patients $\geq 65$ years because aging is one of the risk factors for developing glaucoma. In this study, the glaucoma medical expenditures for the age group over 65 years significantly increased and accounted for more than half the amount of medical expenditures; a reflection of Taiwan's aging population has great financial burden in glaucoma care. Furthermore, we also found that expenditures for glaucoma medications increased significantly for patients in the age group $<40$ years, possibly because of advanced diagnostic tools, more aggressive glaucoma treatment, and the high prevalence of myopia among younger people in Taiwan $[5,15,20]$.

The study results show that blue-collar workers have lower glaucoma medical expenditures, while higher income families incur higher glaucoma medical expenditures, indicating inequality in health care services under universal health coverage in Taiwan. Such inequality may be the result of differences in general knowledge of health care, out-ofpocket payment policy, and other socioeconomic disparities $[10,21,22]$. 
The total expenditure for $\beta$-blockers decreased because of the reduction in cost by the bureau of $\mathrm{NHI}$ and the decrease in usage of $\beta$-blockers, mainly due to the increased availability of PGAs and $\alpha$-agonists. However, considering the drug price, the Taiwan NHI stipulates that $\beta$-blockers are firstline medications and PGAs, CAIs, and fixed combinations are second-line medications. Therefore, $\beta$-blockers still have a considerable market share in Taiwan, despite the fact that PGAs are more effective, are associated with fewer adverse effects, require only once-a-day dosing, and are associated with greater patient compliance [23]. The medical expenditures in the USA have also undergone similar changes relative to insurance coverage [8].

Cholinergic agonists are mainly used for ACG. Even though most people in Taiwan are Chinese in origin and have a higher prevalence of ACG $[2,17]$, there has been a decreasing trend in the application of cholinergic agonists, which may be offset by the increased availability of other drugs like PGAs and the increase in frequency of cataract surgery in Taiwan $[13,23]$. The trend in increasing expenditures for fixed combinations of medications during the period of study can be attributed to patients' preference, because the fixed combinations improve medical adherence and reduce eye discomfort [6].

Trabeculectomy is the most common glaucoma surgery in Taiwan. Our study reveals that the number of trabeculectomies decreased significantly in 1999 and 2000, at the time when PGAs were launched and the Taiwan NHI began to reimburse expenses for PGAs. A similar situation of decreasing the number of trabeculectomies after PGAs listing was also reported in Scotland, France, and Australia [16]. Trabeculectomy is more effective in reducing IOP and lowering diurnal tension than PGAs [24]. However, the side effects of trabeculectomy are greater than those associated with PGAs. According to Cutler and McClellan [25], technological change affects treatment in two ways-treatment substitution and treatment expansion or both. Treatment substitution implies a new technology in place of an old one. Treatment expansion takes place when treatments become safer and easier, and patients pay more attention to their conditions when therapy is more effective or less side effective. Based on the results of this study, we presume that PGAs may have the effect of treatment expansion for trabeculectomy under universal health coverage.

There are several limitations to this study. First of all, the study ignored patient adherence and disease severity. Poor patient adherence may reduce medication expenditures at first but eventually will increase medical and surgical expenditures due to symptom complications. Realistically, severities of glaucoma are related to medical expenditures. Secondarily, insured salary is not necessarily truly representative of patients' socioeconomic status. Most people are employees and pay the insurance fee according to their salary. However, the health insurance fee is lower for those living on their investments. We cannot definitely determine the interaction between socioeconomic status and glaucoma medical expenditures. Thirdly, we did not characterize which types of glaucoma and which education levels contributed to medical expenditures.

\section{Conclusions}

This nationwide population-based study demonstrated an increasing trend in glaucoma medical expenditures from 1997 to 2010 in Taiwan. The main factors contributing to these trends include administration of brand-name drug products such as PGAs, the increasing glaucoma population, good medical accessibility, and possibly more aggressive treatment. PGAs may have the effect of treatment expansion for trabeculectomy. Expenditures were highest among men, patients over 40 years of age, and patients with higher incomes and were lowest among blue-collar workers. The inequality of health care in different socioeconomic disparities may still exist in Taiwan.

\section{Conflict of Interests}

The authors declare that there is no conflict of interests regarding the publication of this paper.

\section{References}

[1] H. A. Quigley and A. T. Broman, "The number of people with glaucoma worldwide in 2010 and 2020," The British Journal of Ophthalmology, vol. 90, no. 3, pp. 262-267, 2006.

[2] J.-W. Cheng, S.-W. Cheng, X.-Y. Ma, J.-P. Cai, Y. Li, and R.-L. Wei, "The prevalence of primary glaucoma in mainland China: a systematic review and meta-analysis," Journal of Glaucoma, vol. 22, no. 4, pp. 301-306, 2013.

[3] A. L. Coleman and S. Miglior, "Risk factors for glaucoma onset and progression," Survey of Ophthalmology, vol. 53, no. 6, pp. S3-S10, 2008.

[4] M. W. Marcus, M. M. de Vries, F. G. J. Montolio, and N. M. Jansonius, "Myopia as a risk factor for open-angle glaucoma: a systematic review and meta-analysis," Ophthalmology, vol. 118, no. 10, pp. 1989.e2-1994.e2, 2011.

[5] NICE, Diagnosis and Management of Chronic Open Angle Glaucoma and Ocular Hypertension, 2014, https://www.nice.org.uk/ guidance/cg85/resources/guidance-glaucoma-pdf.

[6] R. D. Fechtner, D. G. Godfrey, D. Budenz, J. A. Stewart, W. C. Stewart, and M. C. Jasek, "Prevalence of ocular surface complaints in patients with laucoma using topical intraocular pressure-lowering medications," Cornea, vol. 29, no. 6, pp. 618621, 2010.

[7] M. Dirani, J. G. Crowston, P. S. Taylor et al., "Economic impact of primary open-angle glaucoma in Australia," Clinical \& Experimental Ophthalmology, vol. 39, no. 7, pp. 623-632, 2011.

[8] B. L. Lam, D. D. Zheng, E. P. Davila et al., "Trends in glaucoma medication expenditure: medical expenditure panel survey 2001-2006," Archives of Ophthalmology, vol. 129, no. 10, pp. 1345-1350, 2011.

[9] J. Olsen, G. Berdeaux, and J. Skov, "Glaucoma costs in Denmark in treatment naive patients," Acta Ophthalmologica, vol. 91, no. 1, pp. 25-31, 2013.

[10] NHIT: Universal Health Coverage in Taiwan, 2014, http://www .nhi.gov.tw/Resource/webdata/21717_1_20120808UniversalHealthCoverage.pdf.

[11] K. Davis and A. T. Huang, "Learning from Taiwan: experience with universal health insurance," Annals of Internal Medicine, vol. 148, no. 4, pp. 313-314, 2008. 
[12] U. E. Reinhardt, "Humbled in Taiwan," British Medical Journal, vol. 336, no. 7635, article 72, 2008.

[13] C.-C. Hu, H.-C. Lin, C.-S. Chen, and N.-W. Kuo, "Reduction in admissions of patients with acute primary angle closure occurring in conjunction with a rise in cataract surgery in Taiwan," Acta Ophthalmologica, vol. 86, no. 4, pp. 440-445, 2008.

[14] G. C. Chow, "Tests of equality between sets of coefficients in two linear regressions," Econometrica, vol. 28, no. 3, pp. 591-605, 1960.

[15] D. S. Grewal and A. P. Tanna, "Diagnosis of glaucoma and detection of glaucoma progression using spectral domain optical coherence tomography," Current Opinion in Ophthalmology, vol. 24, no. 2, pp. 150-161, 2013.

[16] A. Azuara-Blanco and J. Burr, "The rising cost of glaucoma drugs," The British Journal of Ophthalmology, vol. 90, no. 2, pp. 130-131, 2006.

[17] N. G. Congdon, H. A. Quigley, P. T. Hung, T. H. Wang, and T. C. Ho, "Screening techniques for angle-closure glaucoma in rural Taiwan," Acta Ophthalmologica Scandinavica, vol. 74, no. 2, pp. 113-119, 1996.

[18] T. S. Vajaranant, S. Nayak, J. T. Wilensky, and C. E. Joslin, "Gender and glaucoma: what we know and what we need to know," Current Opinion in Ophthalmology, vol. 21, no. 2, pp. 9199, 2010.

[19] D. S. C. Lam, C. C. Y. Tham, J. S. M. Lai, and D. Y. L. Leung, "Current approaches to the management of acute primary angle closure," Current Opinion in Ophthalmology, vol. 18, no. 2, pp. 146-151, 2007.

[20] Y.-H. Guo, H.-Y. Lin, L. L. K. Lin, and C.-Y. Cheng, "Selfreported myopia in Taiwan: 2005 Taiwan National Health Interview Survey," Eye, vol. 26, no. 5, pp. 684-689, 2012.

[21] J. G. M. M. Hoevenaars, J. S. A. G. Schouten, B. van den Borne, H. J. M. Beckers, and C. A. B. Webers, "Socioeconomic differences in glaucoma patients' knowledge, need for information and expectations of treatments," Acta Ophthalmologica Scandinavica, vol. 84, no. 1, pp. 84-91, 2006.

[22] Y. M. Buys and Y.-P. Jin, "Canadian Glaucoma Risk Factor Study G: socioeconomic status as a risk factor for late presentation of glaucoma in Canada," Canadian Journal of Ophthalmology, vol. 48, no. 2, pp. 83-87, 2013.

[23] G. Reardon, G. F. Schwartz, and E. Mozaffari, "Patient persistency with pharmacotherapy in the management of glaucoma," European Journal of Ophthalmology, vol. 13, supplement 4, pp. S44-S52, 2003.

[24] K. Mansouri, S. Orguel, A. Mermoud et al., "Quality of diurnal intraocular pressure control in primary open-angle patients treated with latanoprost compared with surgically treated glaucoma patients: a prospective trial," The British Journal of Ophthalmology, vol. 92, no. 3, pp. 332-336, 2008.

[25] D. M. Cutler and M. McClellan, "Is technological change in medicine worth it?" Health Affairs, vol. 20, no. 5, pp. 11-29, 2001. 


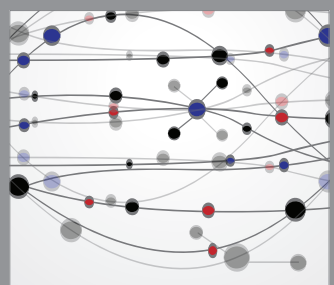

The Scientific World Journal
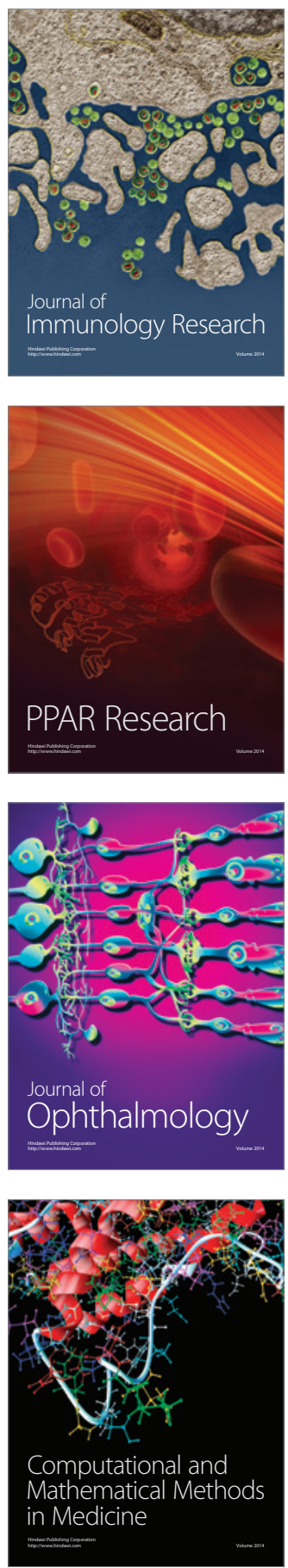

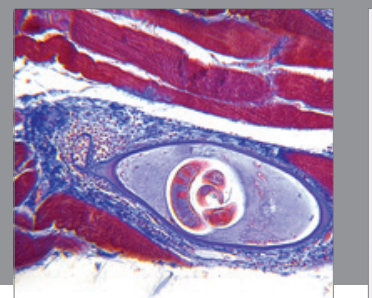

Gastroenterology

Research and Practice
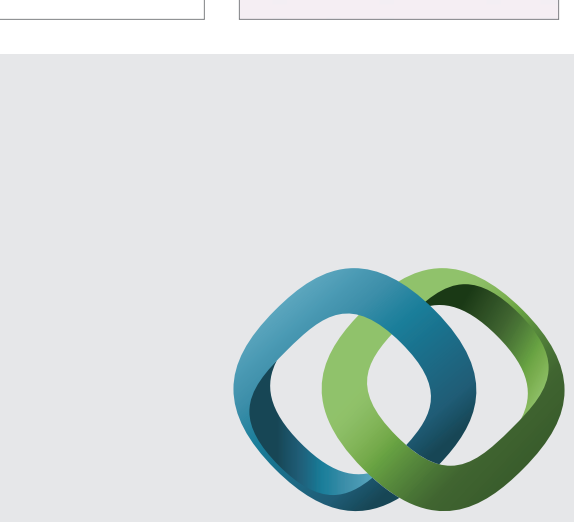

\section{Hindawi}

Submit your manuscripts at

http://www.hindawi.com
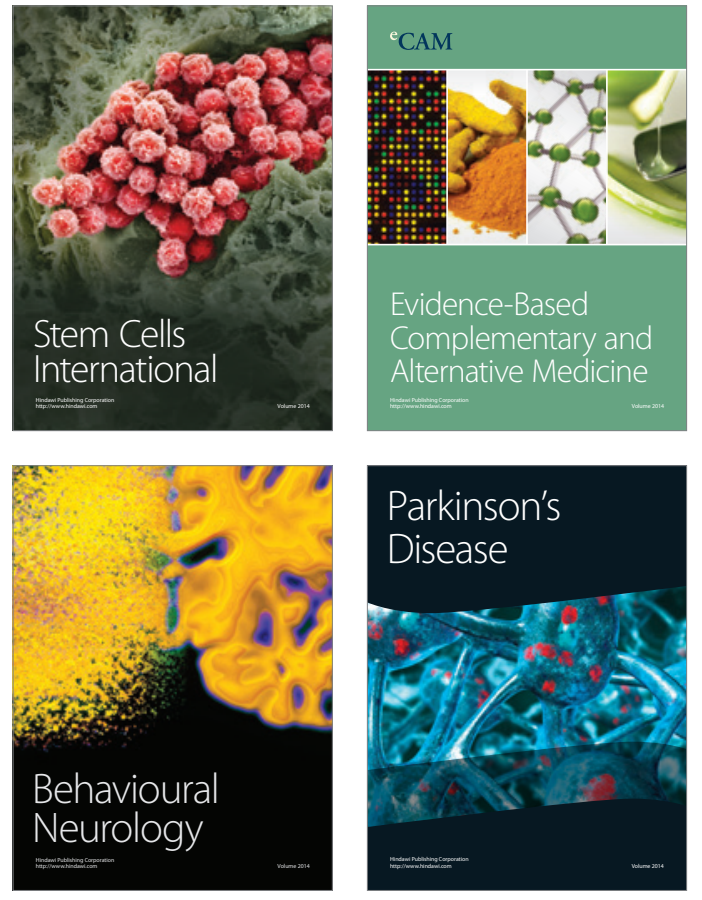
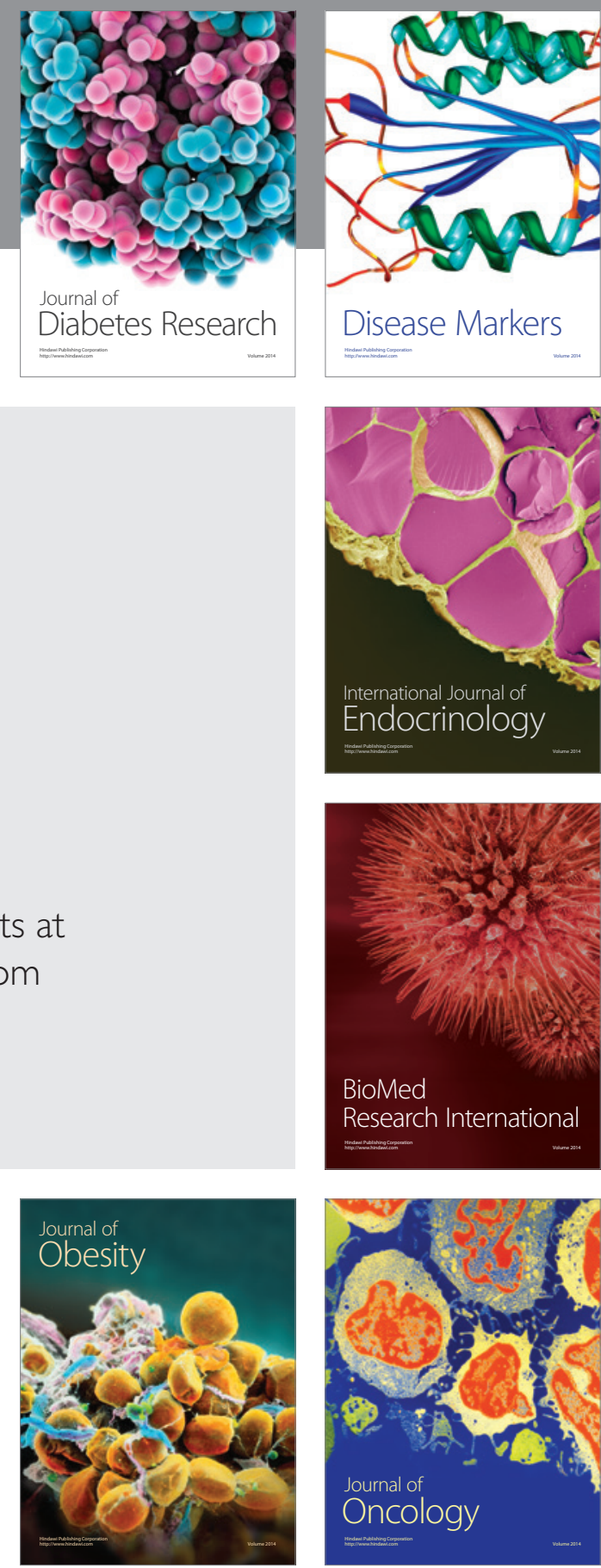

Disease Markers
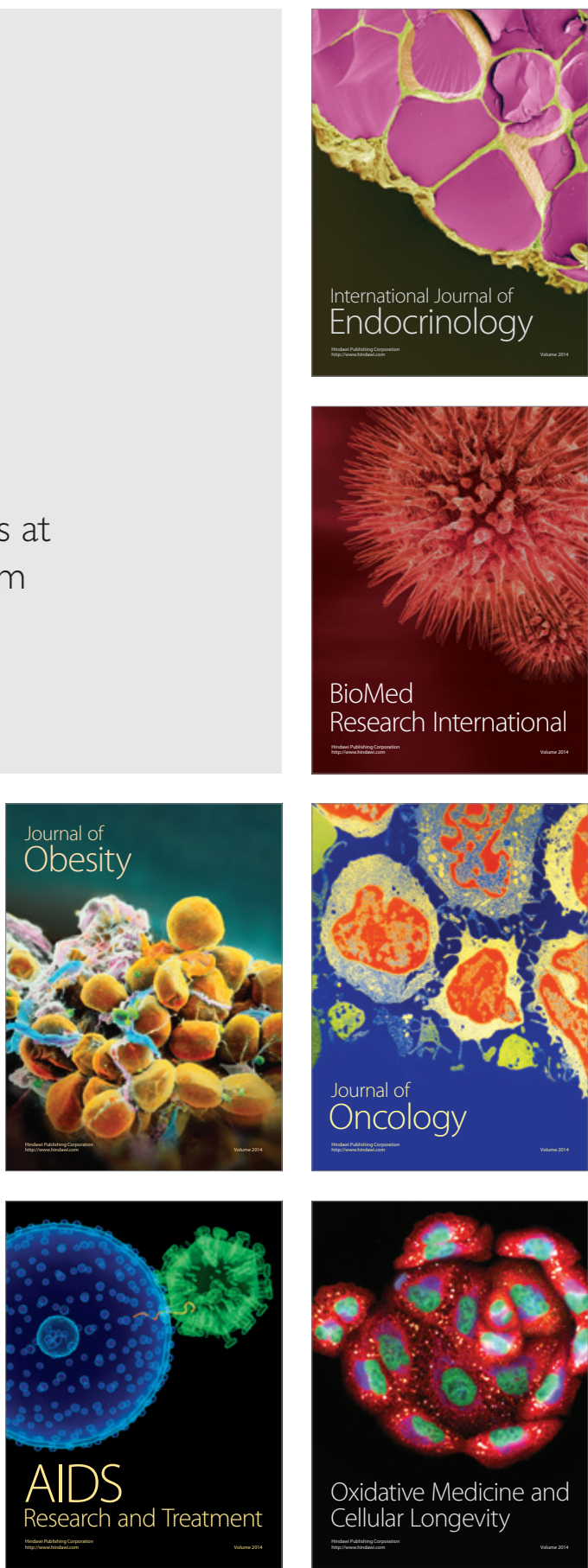